\title{
The 2018 Lake Louise Acute Mountain Sickness Score
}

\author{
Robert C. Roach, ${ }^{1}$ Peter H. Hackett, ${ }^{1}$ Oswald Oelz, ${ }^{2}$ Peter Bärtsch, ${ }^{3}$ Andrew M. Luks, ${ }^{4}$ Martin J. Maclnnis, ${ }^{5}$ \\ J. Kenneth Baillie, ${ }^{6,7}$ and The Lake Louise AMS Score Consensus Committee
}

\begin{abstract}
Roach, Robert C., Peter H. Hackett, Oswald Oelz, Peter Bärtsch, Andrew M. Luks, Martin J. MacInnis, J. Kenneth Baillie, and The Lake Louise AMS Score Consensus Committee. The 2018 Lake Louise Acute Mountain Sickness Score. High Alt Med Biol. 19:4-6, 2018. - The Lake Louise Acute Mountain Sickness (AMS) scoring system has been a useful research tool since first published in 1991. Recent studies have shown that disturbed sleep at altitude, one of the five symptoms scored for AMS, is more likely due to altitude hypoxia per se, and is not closely related to AMS. To address this issue, and also to evaluate the Lake Louise AMS score in light of decades of experience, experts in high altitude research undertook to revise the score. We here present an international consensus statement resulting from online discussions and meetings at the International Society of Mountain Medicine World Congress in Bolzano, Italy, in May 2014 and at the International Hypoxia Symposium in Lake Louise, Canada, in February 2015. The consensus group has revised the score to eliminate disturbed sleep as a questionnaire item, and has updated instructions for use of the score.
\end{abstract}

Keywords: AMS; high altitude illness; history; Lake Louise; symptom scores

Committee members: Eric Achatz, United States; Edi Albert, Australia; Jon S. Andrews, United States; James D. Anholm, United States; Mohammad Zahid Ashraf, India; Paul Auerbach, United States; Buddha Basnyat, Nepal; Beth A. Beidleman, United States; R.R. Berendsen, The Netherlands; Marc Moritz Berger, Austria; Konrad E. Bloch, Switzerland; Hermann Brugger, Italy; Annalisa Cogo, Italy; Ricardo Gonzalez Costa, Argentina; Andrew Cumpstey, United Kingdom; Allen Cymerman, United States; Tadej Debevec, Slovenia; Catriona Duncan, United Kingdom; David Dubowitz, United States; Angela Fago, Denmark; Michael Furian, Switzerland; Matt Gaidica, United States; Prosenjit Ganguli, India; Michael P.W. Grocott, United Kingdom; Debra Hammer, Canada; David Hall, United Kingdom; David Hillebrandt, United Kingdom; Matthias Peter Hilty, Switzerland; Gigugu Himashree, India; Benjamin Honigman, United States; Ned Gilbert-Kawai, United Kingdom; Bengt Kayser, Switzerland; Linda Keyes, United States; Michael Koehle, Canada; Samantha Kohli, India; Arlena Kuenzel, United Kingdom; Benjamin D. Levine, United States; Mona Lichtblau, Switzerland; Jamie Macdonald, United Kingdom; Monika Brodmann Maeder, Italy; Marco Maggiorini, Switzerland; Daniel Martin, United Kingdom; Shigeru Masuyama, Japan; John McCall, Canada; Scott McIntosh, United States; Gregoire Millet, Switzerland; Fernando Moraga, Chile; Craig Mounsey, United Kingdom; Stephen R. Muza, United States; Samuel Oliver, United Kingdom; Qadar Pasha, India; Ryan Paterson, United States; Lara Phillips, United States; Aurélien Pichon, France; Philipp A. Pickerodt, Germany; Matiram Pun, Canada; Manjari Rain, India; Drummond Rennie, United States; Ge Ri-Li, P.R. China; Steven Roy, Canada; Samuel Verges, France; Tatiana Batalha Cunha dos Santos, Brazil; Robert B. Schoene, United States; Otto D. Schoch, Switzerland; Surinderpal Singh, India; Talant Sooronbaev, Kyrgyz Republic; Craig D. Steinback, Canada; Mike Stembridge, United Kingdom; Glenn Stewart, United States; Tsering Stobdan, United States; Giacomo Strapazzon, Italy; Andrew W. Subudhi, United States; Erik Swenson, United States; A. A. Roger Thompson, United Kingdom; Martha Tissot van Patot, United States; Rosie Twomey, Canada; Silvia Ulrich, Switzerland; Nicolas Voituron, France; Dale R. Wagner, United States; Shih-hao Wang, Taiwan; John B. West, United States; Matt Wilkes, United Kingdom; Gabriel Willmann, Germany; Michael Yaron, United States; Ken Zafren, United States.

\footnotetext{
${ }^{1}$ Altitude Research Center, Division of Pulmonary Sciences and Critical Care Medicine, Department of Medicine, University of Colorado School of Medicine, Anschutz Medical Campus, Aurora, Colorado.

${ }^{2}$ Department of Internal Medicine, University of Zurich, Switzerland.

${ }^{3}$ Department of Internal Medicine, University Hospital, Heidelberg, Germany.

${ }^{4}$ Division of Pulmonary, Critical Care and Sleep Medicine, Department of Medicine, University of Washington, Seattle, WA.

${ }^{5}$ Department of Kinesiology, McMaster University, Hamilton, Canada.

${ }^{6}$ Roslin Institute, University of Edinburgh, Easter Bush, Midlothian, United Kingdom.

${ }^{7}$ Intensive Care Unit, Royal Infirmary Edinburgh, Edinburgh, United Kingdom.
} 


\section{Introduction}

A CUTE MOUNTAIN SICKNESS (AMS) is the most common form of acute altitude illness and typically occurs in unacclimatized persons ascending to altitudes $>2500 \mathrm{~m}$, although it can develop at lower altitudes in highly susceptible individuals. Established risk factors include rate of ascent, altitude reached, and individual predisposition. With 25 years of use in hundreds of publications, the Lake Louise AMS score has provided a robust and practical tool for researchers to diagnose and to score the severity of AMS. Recent opinion (Milledge, 2014) and research (MacInnis et al., 2013; Hall et al., 2014) have suggested that updating the Lake Louise AMS score is in order. This article outlines the brief historical background, reviews diagnostic criteria, describes modifications to the score, and offers suggested experimental procedures that may improve the use of the score in future studies.

\section{Background}

At the 1991 International Hypoxia Symposium, the participants executed a consensus process chaired by Peter Hackett and Oswald Oelz (Hackett et al., 1992; supplementary reprint of original article is available online at www.liebertpub.com/ham) to define and quantify the various altitude illnesses. Subsequently at the 1993 conference, all delegates were given the opportunity to have input into the preparation of the document. The score for AMS consisted of the five symptoms (headache, gastrointestinal upset, fatigue/weakness, dizziness/light-headedness, and sleep disturbance), rated on a scale of severity from 0 to 3 . The doubleworded terms were to facilitate understanding as well as translation into many languages (Roach et al., 1993; supplementary reprint of original article is available online at www.liebertpub. $\mathrm{com} / \mathrm{ham})$. A total score $\geq 3$, in the presence of a headache, was considered diagnostic for AMS.

\section{Methods}

This effort is the result of online discussions and meetings at the International Society of Mountain Medicine World Congress in Bolzano, Italy, in May 2014 and at the International Hypoxia Symposium in Lake Louise, Canada, in February 2015. Members of the consensus committee are those who have participated in the online or in-person discussions and are listed in alphabetical order in the box.

\section{Rationale for Revising the Lake Louise AMS Score}

Although use of the scoring system has helped standardize the diagnosis and severity of AMS across research studies, debate has persisted since its inception regarding whether sleep should be included in the diagnostic criteria. Recently this discussion has intensified. Two independent reports in 2013 provided empirical evidence that sleep disturbance is discordant from other symptoms of AMS (MacInnis et al., 2013; Hall et al., 2014). Hall et al. (2014) used network analysis of data from 292 research volunteers exposed to altitudes from 3650 to $5200 \mathrm{~m}$ to demonstrate that sleep disturbance correlated poorly with other symptoms of AMS. Importantly, sleep disturbance was absent in $40 \%$ of cases with severe headache, long considered a hallmark of AMS. MacInnis et al. (2013) applied factor analysis to Lake Louise AMS scores of 491 Nepalese pilgrims at $4390 \mathrm{~m}$ and revealed that sleep had only a weak relationship with the other four symptoms in the score. Milledge also expressed doubt as to whether sleep disturbance was a symptom of AMS, or rather an effect of hypoxia per se, based on his own experience with AMS studies (Milledge, 2014). Another problem recognized over time is that many studies of AMS have used only daytime exposures, making the sleep component irrelevant. Without a score for sleep in these studies, comparison with overnight studies is difficult. Based on these concerns, the consensus committee recommends that the sleep component be removed from the Lake Louise AMS score.

\section{Diagnostic Criteria and Assessment of AMS}

AMS is defined as a Lake Louise AMS score total of three or more points from the four rated symptoms, including at least one point from headache in the setting of a recent ascent or gain in altitude (Roach et al., 2011; West, 2011) (Table 1). Some authors have suggested a higher cutoff for diagnosing AMS (Maggiorini et al., 1998; Bärtsch et al., 2004), but the consensus committee believes that by eliminating the sleep question, more people with true AMS will be identified at the threshold of three points, including headache. Sufficient research is lacking to divide the score into severity rankings. For those who wish to do so, we suggest mild AMS as 3-5 points, moderate AMS as 6-9 points, and severe AMS as 1012 points. Although symptoms can develop within 6 hours of gain in altitude, we recommend assessing AMS score only after 6 hours, to avoid confusing AMS with confounding symptoms from travel or responses to acute hypoxia (e.g., vagal responses). If investigators wish to assess the impact of AMS symptoms on overall function at high altitude, the "AMS Clinical Functional Score" is available (Table 1).

Table 1. 2018 Lake Louise Acute Mountain SiCKNESS SCORE

\begin{tabular}{l} 
Headache \\
0-None at all \\
1-A mild headache \\
2-Moderate headache \\
3-Severe headache, incapacitating \\
Gastrointestinal symptoms \\
0- Good appetite \\
1-Poor appetite or nausea \\
2-Moderate nausea or vomiting \\
3-Severe nausea and vomiting, incapacitating \\
Fatigue and/or weakness \\
0-Not tired or weak \\
1-Mild fatigue/weakness \\
2-Moderate fatigue/weakness \\
3-Severe fatigue/weakness, incapacitating \\
Dizziness/light-headedness \\
0-No dizziness/light-headedness \\
1-Mild dizziness/light-headedness \\
2-Moderate dizziness/light-headedness \\
3-Severe dizziness/light-headedness, incapacitating \\
AMS Clinical Functional Score \\
Overall, if you had AMS symptoms, how did they affect \\
your activities? \\
0-Not at all \\
1-Symptoms present, but did not force any change in \\
2- My symptoms forced me to stop the ascent or to go \\
3- -Had to be evacuated to a lower altitude \\
\hline
\end{tabular}


AMS must not be confused with high-altitude cerebral edema (HACE). AMS alone exhibits no neurological findings, and is self-limited. In contrast, HACE, which usually comes on between 24 and 72 hours after a gain in altitude, is characterized by change in mental status and/or ataxia, occurs usually in a person with AMS or high-altitude pulmonary edema, and is a medical emergency (Hackett and Roach, 2004; Willmann et al., 2014).

\section{Directions for Using the Lake Louise AMS Score}

This Lake Louise AMS score is for use by investigators studying AMS. It is not intended for use by clinicians, professional outdoor guides, and laypersons to diagnose or manage AMS. After a recent gain in altitude or induction of hypoxia, and an exposure of at least 6 hours duration, the AMS score is used as follows:

1. The Lake Louise AMS score is designed as a selfreport questionnaire that research volunteers complete on their own. However, some investigators prefer to read the question to the volunteer and record the answers, whereas others use a two-step method wherein the volunteer first completes the score, then the investigator verbally verifies the answers. These options are acceptable as long as a uniform approach is used with all subjects in a study and the method of collecting data is clearly described in subsequent reports.

2. The Lake Louise AMS score for an individual is the sum of the score for the four symptoms (headache, nausea/ vomiting, fatigue, and dizziness/light-headedness). For a positive AMS definition, it is mandatory to have a headache score of at least one point, and a total score of at least three points.

Example 1: A total score greater than two points but with no headache is defined as NO AMS for research purposes, although absence of a headache does not exclude a diagnosis for clinical purposes.

Example 2: A score of three points for a severe headache, with no other AMS symptoms, is defined as AMS.

3. We suggest using the AMS clinical functional score and reporting it when suitable to the study design (Roach et al., 1993; supplementary reprint of original article is available online at www.liebertpub.com/ham; Meier et al., 2017).

\section{Avenues for Future Research}

Further research should focus on the following areas: (1) best methods for Lake Louise AMS score administration; that is, is investigator-led scoring different/better than volunteercompleted scores? (2) the impact of experimental design, the testing environment, and expectations of research volunteers (i.e., nocebo) (Benedetti et al., 2014) on reliability of Lake Louise AMS score; (3) the clinical and functional impact(s) of AMS score severity; (4) best practices for use of the Lake Louise AMS score and clinical functional score by nonexpert clinicians, mountain guides, and laypersons (Roach et al., 1993; supplementary reprint of original article is available online at www .liebertpub.com/ham; Meier et al., 2017); (5) the impact of disturbed sleep on overall well-being at high altitude, independent of AMS; and (6) the pathophysiology of typical AMS vs. presentation without headache (Roach et al., 2011; West, 2011). In addition, we strongly encourage researchers to publish all in- dividual scores for all volunteers and all symptoms. This will allow other researchers to directly compare patterns of illness, to compile meta-analyses, and to examine the raw data for ideas and observations that will further refine the consensus definition and scoring of AMS.

\section{Author Disclosure Statement}

No competing financial interests exist.

\section{References}

Bärtsch P, Bailey DM, Berger MM, Knauth M, and Baumgartner RW. (2004). Acute mountain sickness: Controversies and advances. High Alt Med Biol 5:110-124.

Benedetti F, Durando J, and Vighetti S. (2014). Nocebo and placebo modulation of hypobaric hypoxia headache involves the cyclooxygenase-prostaglandins pathway. Pain 155:921-928.

Hackett PH, and Oelz O. (1992). The Lake Louise Consensus on the definition and quantification of altitude illness. In: Hypoxia and Mountain Medicine. JR Sutton, CS Houston, G Coates, eds. Queen City Press, Burlington, VT. pp 327-330.

Hackett PH, and Roach RC. (2004). High altitude cerebral edema. High Alt Med Biol 5:136-146.

Hall DP, MacCormick IJ, Phythian-Adams AT, Rzechorzek NM, Hope-Jones D, Cosens S, Jackson S, Bates MG, Collier DJ, Hume DA, et al. (2014). Network analysis reveals distinct clinical syndromes underlying acute mountain sickness. PLoS One 9:e81229.

MacInnis MJ, Lanting SC, Rupert JL, and Koehle MS. (2013). Is poor sleep quality at high altitude separate from acute mountain sickness? Factor structure and internal consistency of the Lake Louise Score Questionnaire. High Alt Med Biol 14:334-337.

Maggiorini M, Muller A, Hofstetter D, Bärtsch P, and Oelz O. (1998). Assessment of acute mountain sickness by different score protocols in the Swiss Alps. Aviat Space Environ Med 69:1186-1192.

Meier D, Collet TH, Locatelli I, Cornuz J, Kayser B, Simel DL, and Sartori C. (2017). Does this patient have acute mountain sickness?: The rational clinical examination systematic review. JAMA 318:1810-1819.

Milledge JS. (2014). Should sleep quality be part of the Lake Louise Acute Mountain Sickness Score? High Alt Med Biol 15:208.

Roach RC, Bärtsch P, Hackett PH, and Oelz O. (1993). The Lake Louise Acute Mountain Sickness Scoring System. In: Hypoxia and Molecular Medicine. JR Sutton, CS Houston, G Coates, eds. Queen City Press, Burlington, VT. pp 272-274.

Roach RC, Kayser B, and Hackett PH. (2011). Pro: Headache should be a required symptom for the diagnosis of acute mountain sickness. High Alt Med Biol 12:21-22.

West JB. (2011). Con: Headache should not be a required symptom for the diagnosis of acute mountain sickness. High Alt Med Biol 12:23-25.

Willmann G, Gekeler F, Schommer K, and Bärtsch P. (2014). Update on high altitude cerebral edema including recent work on the eye. High Alt Med Biol 15:112-122.

Address correspondence to:

Robert C. Roach, PhD

Altitude Research Center

Division of Pulmonary Sciences and Critical Care Medicine

Department of Medicine

Anschutz Medical Campus

University of Colorado School of Medicine Aurora CO 80045

E-mail: rroach@hypoxia.net 